\title{
在 $\left(\boldsymbol{L}_{p}\left(\mathbb{R}^{s}\right)\right)^{r}(\mathbf{1} \leqslant \boldsymbol{p} \leqslant \infty)$ 中的向量细分格式 ${ }^{*}$
}

\author{
李松
}

(浙江大学数学系, 杭州 310027 )

摘要研究如下形式的细分方程:

$$
\varphi(x)=\sum_{\alpha \in \mathbb{Z}^{s}} a(\alpha) \varphi(M x-\alpha), \quad x \in \mathbb{R}^{s},
$$

其中向量值函数 $\varphi=\left(\varphi_{1}, \cdots, \varphi_{r}\right)^{\mathrm{T}} \in\left(L_{p}\left(\mathbb{R}^{s}\right)\right)^{r}(1 \leqslant p \leqslant \infty), a(\alpha)$ 是具有有限长的 $r \times r$ 矩阵值序列, 称为面具, $M$ 是一个 $s \times s$ 整数矩阵, 并且满足 $\lim _{n \rightarrow \infty} M^{-n}=0$. 选择具有紧支集的向量值函数 $\varphi_{0} \in\left(L_{p}\left(\mathbb{R}^{s}\right)\right)^{r}(1 \leqslant p \leqslant \infty)$, 定义

$$
f_{n}:=Q_{a}^{n} \varphi_{0}, \quad n=1,2, \cdots,
$$

其中

$$
Q_{a} \varphi(x)=\sum_{\alpha \in \mathbb{Z}^{s}} a(\alpha) \varphi(M x-\alpha), \quad \varphi \in\left(L_{p}\left(\mathbb{R}^{s}\right)\right)^{r} .
$$

函数列 $\left\{f_{n}\right\}_{n} \geqslant 0$ 称为细分格式或级联序列. 利用由扩张矩阵 $M$, 面具 $a(\alpha)$ 以及集合 $B$ 生成的有限个线性算子的联合谱半径来刻画序列 $\left\{f_{n}\right\}_{n \geqslant 0}$ 在 $\left(L_{p}\left(\mathbb{R}^{s}\right)\right)^{r}(1 \leqslant p \leqslant \infty)$ 中的敛散性, 这里集合 $B$ 表示包含 0 元素的商群 $\mathbb{Z}^{s} / M \mathbb{Z}^{s}$ 的不同代表元.

\section{关键词 细分方程 联合谱半径 细分格式 $\left(\boldsymbol{L}_{\boldsymbol{p}}\left(\mathbb{R}^{s}\right)\right)^{r}(1 \leqslant \boldsymbol{p} \leqslant \infty)$ 空间}

本文考虑如下形式的函数方程:

$$
\varphi(x)=\sum_{\alpha \in \mathbb{Z}^{s}} a(\alpha) \varphi(M x-\alpha), \quad x \in \mathbb{R}^{s},
$$

其中向量值函数 $\varphi=\left(\varphi_{1}, \cdots, \varphi_{r}\right)^{\mathrm{T}} \in\left(L_{p}\left(\mathbb{R}^{s}\right)\right)^{r}(1 \leqslant p \leqslant \infty), a(\alpha)$ 是具有有限长的 $r \times r$ 矩阵值 序列, $M$ 是 $s \times s$ 整数矩阵, 并且满足 $\lim _{n \rightarrow \infty} M^{-n}=0$. 方程 (0.1) 称为齐次细分方程, $a$ 称为 面具, 矩阵 $M$ 称为扩张矩阵. 众所周知, 满足方程 $(0.1)$ 的函数称为细分函数. 设 $1 \leqslant p \leqslant \infty$, 用 $\left(L_{p}\left(\mathbb{R}^{s}\right)\right)^{r}$ 表示所有满足 $\|f\|_{p}<\infty$ 的向量值函数构成的 Banach 空间，其中

$$
\|f\|_{p}:=\left(\sum_{j=1}^{r} \int_{\mathbb{R}^{s}}\left|f_{j}\right|^{p} d x\right)^{\frac{1}{p}}, \quad 1 \leqslant p<\infty,
$$

$\|f\|_{\infty}=\operatorname{ess}_{\sup } \in \mathbb{R}^{s} \max _{1 \leqslant j \leqslant r}\left|f_{j}\right|$.

2002-11-22 收稿

* 国家自然科学基金资助项目（批准号: 10071071) 
对一个可积向量值函数 $f$, 定义它的 Fourier 变换 $\hat{f}$ 为

$$
\hat{f}(\xi):=\int_{\mathbb{R}^{s}} f(x) e^{-\mathrm{i} x \cdot \xi} d x, \quad \xi \in \mathbb{R}^{s},
$$

其中 $x \cdot \xi$ 表示向量 $x$ 与 $\xi$ 在 $\mathbb{R}^{s}$ 中的内积.

对方程 (0.1) 两端取 Fourier 变换, 得到

$$
\hat{\varphi}(\xi)=H\left(\left(M^{\mathrm{T}}\right)^{-1} \xi\right) \hat{\varphi}\left(\left(M^{\mathrm{T}}\right)^{-1} \xi\right), \quad \xi \in \mathbb{R}^{s},
$$

这里 $M^{\mathrm{T}}$ 表示 $M$ 的转置矩阵,

$$
H(\xi):=\frac{1}{|\operatorname{det} M|} \sum_{\alpha \in \mathbb{Z}^{s}} a(\alpha) e^{-\mathrm{i} \alpha \cdot \xi}, \quad \xi \in \mathbb{R}^{s} .
$$

容易看到若 $\hat{\varphi}(0)$ 不是零向量, 则向量 $\hat{\varphi}(0)$ 是矩阵 $H(0)$ 对应于特征值 1 的特征向量.

设 $A$ 是一个矩阵 (或者 $A$ 是定义在有限维线性空间上的线性算子), 用符号 $\rho(A)$ 来定义 $A$ 的谱半径. 如果 $\rho(A) \leqslant 1$, 并且 1 是 $A$ 在单位圆周上的惟一的简单特征值, 则称 $A$ 满足条 件 $E$. 设 $\varphi=\left(\varphi_{1}, \cdots, \varphi_{r}\right)^{\mathrm{T}} \in\left(L_{1}\left(\mathbb{R}^{s}\right)\right)^{r}$ 是紧支集并且满足方程 $(0.1)$, 文献 [1,2] 证明了: 如果 $\hat{\varphi}(0)$ 不是零向量, 并且 $\operatorname{span}\left\{\hat{\varphi}(2 \pi \beta): \beta \in \mathbb{Z}^{s}\right\}=\mathbb{C}^{r}$, 则 $H(0)$ 满足条件 $E$. 本文总假设 $H(0)$ 满足条件 $E$. 这样, 存在一个可逆矩阵 $U$, 使得 $U H(0) U^{-1}$ 具有形式

$$
\left(\begin{array}{ll}
1 & 0 \\
0 & \Lambda
\end{array}\right),
$$

其中 $\Lambda$ 是一个 $(r-1) \times(r-1)$ 矩阵并且满足 $\lim _{n \rightarrow \infty} \Lambda^{n}=0$. 记 $b(\alpha)=U a(\alpha) U^{-1}$, 如果 $\varphi$ 是 细分方程 $(0.1)$ 的解, 则 $\psi=U \varphi$ 满足细分方程

$$
\psi=\sum_{\alpha \mathbb{Z}^{s}} b(\alpha) \psi(M \cdot-\alpha), \quad x \in \mathbb{R}^{s},
$$

因此不失一般性, 总可以假设 $H(0)$ 具有 $(0.5)$ 式的形式.

设 $j=1,2, \cdots, r$, 用 $e_{j}$ 表示 $r$ 阶单位矩阵的第 $j$ 列向量. 利用 (0.5) 式，有

$$
e_{1}^{\mathrm{T}} H(0)=e_{1}^{\mathrm{T}} \text {. }
$$

假设 $H(0)$ 具有 $(0.5)$ 式的形式, 文献 [3] 证明了方程 (0.1) 存在惟一紧支集规范化分布解 $\varphi(x)$, 即 $\varphi(x)$ 满足 $\hat{\varphi}(0)=(1,0, \cdots, 0)^{\mathrm{T}}$, 文献 [4] 给出了方程 $(0.1)$ 具有 $L_{2^{-}}$解的充分必要条 件.

为了解细分方程 $(0.1)$, 引入线性算子 $Q_{a}$,

$$
Q_{a} \varphi(x)=\sum_{\alpha \in Z^{s}} a(\alpha) \varphi(M x-\alpha), \quad x \in \mathbb{R}^{s} .
$$

利用归纳法容易证明

$$
Q_{a}^{n} \varphi(x)=\sum_{\alpha \in \mathbb{Z}^{s}} a_{n}(\alpha) \varphi\left(M^{n} x-\alpha\right),
$$

其中 $a_{1}=a, a_{n}$ 由如下递推式给出:

$$
a_{n}(\alpha) \varphi=\sum_{\beta \in \mathbb{Z}^{s}} a_{n-1}(\beta) a(\alpha-M \beta), \quad \alpha \in \mathbb{Z}^{s} .
$$

设 $f \in\left(L_{p}\left(\mathbb{R}^{s}\right)\right)^{r}$ 是一个紧支集向量值函数, $Q_{a}^{n} f$ 在 $\left(L_{p}\left(\mathbb{R}^{s}\right)\right)^{r}(1 \leqslant p \leqslant \infty)$ 中收玫到方程 $(0.1)$ 的规范化解 $\varphi(x)$. 利用 $(0.7)$ 式得到

$$
\hat{Q_{a}} f(\xi)=H\left(\left(M^{\mathrm{T}}\right)^{-1} \xi\right) \hat{f}\left(\left(M^{\mathrm{T}}\right)^{-1} \xi\right), \quad \xi \in \mathbb{R}^{s} .
$$


由 $(0.10)$ 式有

$$
Q_{a}^{\hat{n}} f(\xi)=\prod_{k=1}^{n} H\left(\left(M^{\mathrm{T}}\right)^{-k} \xi\right) \hat{f}\left(\left(M^{\mathrm{T}}\right)^{-n} \xi\right), \quad \xi \in \mathbb{R}^{s} .
$$

特别对上式取 $\beta \in \mathbb{Z}^{s}$, 推得

$$
Q_{a}^{\hat{n}} f\left(2 \pi\left(M^{\mathrm{T}}\right)^{n} \beta\right)=H(0)^{n} \hat{f}(2 \pi \beta)
$$

因此,

$$
e_{1}^{\mathrm{T}} \hat{Q}_{a}^{\hat{n}} f\left(2 \pi\left(M^{\mathrm{T}}\right)^{n} \beta\right)=e_{1}^{\mathrm{T}} H(0)^{n} \hat{f}(2 \pi \beta)=e_{1}^{\mathrm{T}} \hat{f}(2 \pi \beta) .
$$

因为 $Q_{a}^{n} f$ 在 $\left(L_{p}\left(\mathbb{R}^{s}\right)\right)^{r}(1 \leqslant p \leqslant \infty)$ 中收玫到方程 $(0.1)$ 的规范化解 $\varphi(x)$, 利用 RiemannLebesgue 引理, 有

$$
\lim _{n \rightarrow \infty} \hat{Q}_{a}^{\hat{n}} f\left(2 \pi\left(M^{\mathrm{T}}\right)^{n} \beta\right)=\lim _{n \rightarrow \infty} \hat{\varphi}\left(2 \pi\left(M^{\mathrm{T}}\right)^{n} \beta\right)=\hat{\varphi}(0), \quad \beta=0
$$

及

$$
\lim _{n \rightarrow \infty} Q_{a}^{\hat{n}} f\left(2 \pi\left(M^{\mathrm{T}}\right)^{n} \beta\right)=\lim _{n \rightarrow \infty} \hat{\varphi}\left(2 \pi\left(M^{\mathrm{T}}\right)^{n} \beta\right)=0, \quad \beta \neq 0
$$

因此

$$
e_{1}^{\mathrm{T}} \hat{f}(0)=\lim _{n \rightarrow \infty} e_{1}^{\mathrm{T}} Q_{a}^{\hat{n}} f(0)=e_{1}^{\mathrm{T}} \hat{\varphi}(0)=1
$$

及

$$
e_{1}^{\mathrm{T}} \hat{f}(2 \pi \beta)=\lim _{n \rightarrow \infty} e_{1}^{\mathrm{T}} \hat{Q_{a}^{n}} f\left(2 \pi\left(M^{\mathrm{T}}\right)^{n} \beta\right)=0, \quad \beta \in \mathbb{Z}^{s} \backslash\{0\} .
$$

由 Poisson 求和公式知 $(0.11)$ 和 $(0.12)$ 式等价于

$$
e_{1}^{\mathrm{T}} \sum_{\alpha \in \mathbb{Z}^{s}} f(\cdot-\alpha)=1,
$$

因此, 如果存在一个向量值函数 $\varphi \in\left(L_{p}\left(\mathbb{R}^{s}\right)\right)^{r}(1 \leqslant p \leqslant \infty)$, 使得对任意满足条件 $(0.13)$ 的紧 支集向量值函数 $f \in\left(L_{p}\left(\mathbb{R}^{s}\right)\right)^{r}(1 \leqslant p \leqslant \infty)$ 都有

$$
\lim _{n \rightarrow \infty}\left\|Q_{a}^{n} f-\varphi\right\|_{p}=0,
$$

则称与面具 $a$ 以及扩张矩阵 $M$ 有关的细分格式 (或级联序列) 在 $\left(L_{p}\left(\mathbb{R}^{s}\right)\right)^{r}(1 \leqslant p \leqslant \infty)$ 中收 敛.

在小波分析理论中, 细分格式的收玫性是基本的 ${ }^{[5 \sim 7]}$. 当 $r=1$ 及 $M=2$ 时, $\mathrm{Dyn}^{[8]}$ 等在 $C(\mathbb{R})$ 中研究了细分格式的收玫性, $\mathrm{Jia}^{[9]}$ 在 $L_{p}(\mathbb{R})(1 \leqslant p \leqslant \infty)$ 中也研究了细分格式的收玫性.

当 $r=1$ 及 $s>1, \operatorname{Han}^{[10]}$ 等研究了当 $M$ 为一般的扩张矩阵时细分格式在 $L_{p}\left(\mathbb{R}^{s}\right)(1 \leqslant p \leqslant \infty)$ 中的收玫性, Strang ${ }^{[11]}$ 和 Latwon ${ }^{[12]}$ 等也分别研究了细分格式在 $L_{2}\left(\mathbb{R}^{s}\right)$ 中的收玫性. 当 $r>1$, $M=2$ 以及 $H(0)$ 具有 $(0.5)$ 式的形式时, $\mathrm{Jia}^{[2]}$ 等与 Long ${ }^{[13]}$ 等分别研究了细分格式在 $\left(L_{p}(\mathbb{R})\right)^{r}$ $(1 \leqslant p \leqslant \infty)$ 及 $\left(L_{2}(\mathbb{R})\right)^{r}$ 中的收玫性. 当 $r>1$ 以及 $M=2 I$ 时, Shen ${ }^{[14]}$ 研究了细分格式在 $\left(L_{2}(\mathbb{R})\right)^{r}$ 中的收玫性.

当扩张矩阵 $M$ 是各向同性时, 即 $M$ 相似于对角矩阵 $\operatorname{diag}\left(\sigma_{1}, \cdots, \sigma_{s}\right)$, 其中 $\left|\sigma_{1}\right|=\cdots=\left|\sigma_{s}\right|$, 文献 $[1,15]$ 也分别研究了细分格式在 Sobolev 空间 $\left(W_{p}^{k}\left(\mathbb{R}^{s}\right)\right)^{r}$ 及 $W_{2}^{k}\left(\mathbb{R}^{s}\right)$ 中的收玫性.

本文的主要目的是利用由扩张矩阵 $M$ 和面具 $a(\alpha)$ 以及集合 $B$ 生成的有限个线性算子的 $p$ - 范数联合谱半径, 来刻画细分格式在 $\left(L_{p}\left(\mathbb{R}^{s}\right)\right)^{r}(1 \leqslant p \leqslant \infty)$ 中的玫散性, 这里集合 $B$ 表示包 
含 0 元素的商群 $\mathbb{Z}^{s} / M \mathbb{Z}^{s}$ 的不同代表元. 我们指出, 所有以上提到的细分格式在 $\left(L_{p}\left(\mathbb{R}^{s}\right)\right)^{r}(1 \leqslant$ $p \leqslant \infty)$ 中以及在 $\left(L_{2}\left(\mathbb{R}^{s}\right)\right)^{r}$ 中收玫性的特征刻画都是本文结果的特殊情形.

\section{1 基本和规则与一些线性算子}

记所有有限长的 $r \times r$ 矩阵值序列的全体为 $\left(l_{0}\left(\mathbb{Z}^{s}\right)\right)^{r \times r}$. 设 $M$ 是一个给定的扩张矩阵, 记 $m=|\operatorname{det} M|$. 我们知道商群 $\mathbb{Z}^{s} / M \mathbb{Z}^{s}$ 包含 $m$ 个元素. 用集合 $B=\left\{\gamma_{k}+M \mathbb{Z}^{s}, k=0,1, \cdots, m-1\right\}$ 表示商群 $\mathbb{Z}^{s} / M \mathbb{Z}^{s}$ 不同的代表元，其中 $\gamma_{0}=0$, 则任意一个整数 $\alpha$ 都能惟一表示为 $\varepsilon+M \gamma$, 其 中 $\varepsilon \in B, \gamma \in \mathbb{Z}^{s}$. 对 $\varepsilon \in B$ 和 $a \in\left(l_{0}\left(\mathbb{Z}^{s}\right)\right)^{r \times r}$, 引入定义在 $\left(l_{0}\left(\mathbb{Z}^{s}\right)\right)^{r \times 1}$ 上的线性算子 $A_{\varepsilon}$,

$$
A_{\varepsilon} u(\alpha):=\sum_{\beta \in \mathbb{Z}^{s}} a(\varepsilon+M \alpha-\beta) u(\beta), \quad \alpha \in \mathbb{Z}^{s}, u \in\left(l_{0}\left(\mathbb{Z}^{s}\right)\right)^{r} .
$$

对 $k=0,1, \cdots, m-1$, 如果

$$
e_{1}^{\mathrm{T}} \sum_{\alpha \in \mathbb{Z}^{s}} a\left(M \alpha+\gamma_{k}\right)=e_{1}^{\mathrm{T}},
$$

则称 $a$ 满足基本和规则. 文献 [4] 证明了如果方程 (0.1) 有一个紧支集稳定解，则由 (0.4) 式确 定的 $H(0)$ 满足 $E$ 条件, 同时 $a$ 满足基本和规则.

定理 1.1 设 $a \in\left(l_{0}\left(\mathbb{Z}^{s}\right)\right)^{r \times r}, H(0)$ 满足 $E$ 条件, $M$ 是一个扩张矩阵. 如果存在一个正 数 $p \geqslant 1$, 使得细分格式在 $\left(L_{p}\left(\mathbb{R}^{s}\right)\right)^{r}$ 中收玫, 则

$$
e_{1}^{\mathrm{T}} \sum_{\alpha \in \mathbb{Z}^{s}} a\left(M \alpha+\gamma_{k}\right)=e_{1}^{\mathrm{T}}, \quad k=0,1, \cdots, m-1 .
$$

证 假设 $f \in\left(L_{p}\left(\mathbb{R}^{s}\right)\right)^{r}$ 是满足 $(0.13)$ 式的紧支集向量值函数. 因为 $\left(Q_{a}^{n} f\right)_{n=1}^{\infty}$ 在 $\left(L_{p}\left(\mathbb{R}^{s}\right)\right)^{r}$ 中收玫, 因此 $\left(Q_{a}^{n}\left(Q_{a} f\right)\right)_{n=1}^{\infty}$ 也在 $\left(L_{p}\left(\mathbb{R}^{s}\right)\right)^{r}$ 中收玫. 由以上的讨论, 有

$$
e_{1}^{\mathrm{T}} \sum_{\alpha \in \mathbb{Z}^{s}} Q_{a} f(\cdot-\alpha)=1 .
$$

利用 $(0.7)$ 式得到

$$
\begin{aligned}
\sum_{\alpha \in \mathbb{Z}^{s}} Q_{a} f(\cdot-\alpha) & =\sum_{\beta \in \mathbb{Z}^{s}} \sum_{\alpha \in \mathbb{Z}^{s}} a(\beta-M \alpha) f(M \cdot-\beta) \\
& =\sum_{k=0}^{m-1} \sum_{\gamma \in \mathbb{Z}^{s}} \sum_{\alpha \in \mathbb{Z}^{s}} a\left(M \gamma-M \alpha+\gamma_{k}\right) f\left(M \cdot-M \gamma-\gamma_{k}\right) .
\end{aligned}
$$

设 $y$ 是一个 $r \times 1$ 的复向量, 并且满足 $e_{1}^{\mathrm{T}} y=1, \varphi_{0}$ 是由下式确定的函数:

$$
\varphi_{0}(x)=\prod_{j=1}^{s} \chi\left(x_{j}\right), \quad x=\left(x_{1}, \cdots, x_{s}\right) \in \mathbb{R}^{s},
$$

其中 $\chi(t)$ 是定义在 $[0,1)$ 上的特征函数. 选择 $f=y \varphi_{0}$, 显然, $y \varphi_{0}$ 满足 $(0.13)$ 式. 对 $\gamma_{j}$, 选择 $x \in \mathbb{R}^{s}$, 使得 $M x-\gamma_{j} \in[0,1)^{s}$, 这样对任意的 $\gamma \in \mathbb{Z}^{s}, k \neq j$, 有 $M x-\gamma_{k}-M \gamma \notin[0,1)^{s}$. 由 (1.5) 式, 得到

$$
\sum_{\alpha \in \mathbb{Z}^{s}} Q_{a} f(\cdot-\alpha)=\sum_{\alpha \in \mathbb{Z}^{s}} a\left(-M \alpha+\gamma_{j}\right) y .
$$

再利用 (1.4) 式, 有

$$
e_{1}^{\mathrm{T}} \sum_{\alpha \in \mathbb{Z}^{s}} a\left(-M \alpha+\gamma_{j}\right) y=1 .
$$


因为上述等式对任意满足 $e_{1}^{\mathrm{T}} y=1$ 的 $y \in \mathbb{C}^{r}$ 都成立, 因此

$$
e_{1}^{\mathrm{T}} \sum_{\alpha \in \mathbb{Z}^{s}} a\left(M \alpha+\gamma_{j}\right)=e_{1}^{\mathrm{T}} \text {. }
$$

证毕.

注 1.1 当 $r=1, p=\infty$ 及 $M=2 I$ 时, Cavaretta 等 ${ }^{[5]}$ 证明了这个结论. 当 $r=1,1 \leqslant p \leqslant$ $\infty$ 及 $M$ 是一般的扩张矩阵时, $\mathrm{Han}$ 和 $\mathrm{Jia}^{[10]}$ 得到了这个结论. 当 $r>1, s=1$ 及 $M=2$ 时, $\mathrm{Jia}$ 等 ${ }^{[2]}$ 也获得了这个结果.

设 $\beta \in \mathbb{Z}^{s}$, 定义序列

$$
\delta_{\beta}(\alpha)= \begin{cases}1, & \text { 当 } \alpha=\beta, \\ 0, & \text { 当 } \alpha \in \mathbb{Z}^{s} \backslash\{\beta\} .\end{cases}
$$

当 $\beta=0$ 时, 记 $\delta$ 为 $\delta_{0}$. 设 $\lambda \in \mathbb{Z}^{s}$, 用 $\nabla_{\lambda}$ 表示由下式定义的差分算子:

$$
\nabla_{\lambda} v=v-v(\cdot-\lambda), \quad \lambda \in l\left(\mathbb{Z}^{s}\right) .
$$

为方便, 记 $\nabla_{j}$ 为 $\nabla_{e_{j}}, j=1, \cdots, s$.

记

$$
V:=\left\{v \in\left(l_{0}\left(\mathbb{Z}^{s}\right)\right)^{r}: e_{1}^{\mathrm{T}} \sum_{\alpha \in \mathbb{Z}^{s}} v(\alpha)=0\right\} .
$$

定理 1.2 设 $a$ 和 $M$ 与定理 1.1 中一致, $A_{\varepsilon}$ 由 (1.1) 式给出, 则对任意 $\varepsilon \in B, V$ 均为 $A_{\varepsilon}$ 的不变线性空间的充分必要条件是

$$
e_{1}^{\mathrm{T}} \sum_{\alpha \in \mathbb{Z}^{s}} a\left(M \alpha+\gamma_{k}\right)=e_{1}^{\mathrm{T}}, \quad k=0,1, \cdots, m-1 .
$$

证 假设 $a$ 满足基本和规则, 并且 $v \in V$, 则对任意的 $\gamma_{k}$, 有

$$
\begin{aligned}
e_{1}^{\mathrm{T}} \sum_{\alpha \in \mathbb{Z}^{s}} A_{\gamma_{k}} v(\alpha) & =e_{1}^{\mathrm{T}} \sum_{\alpha \in \mathbb{Z}^{s}} \sum_{\beta \in \mathbb{Z}^{s}} a\left(M \alpha+\gamma_{k}-\beta\right) v(\beta) \\
& =\sum_{\beta \in \mathbb{Z}^{s}}\left[e_{1}^{\mathrm{T}} \sum_{\alpha \in \mathbb{Z}^{s}} a\left(M \alpha+\gamma_{k}-\beta\right)\right] v(\beta)=\sum_{\beta \in \mathbb{Z}^{s}} e_{1}^{\mathrm{T}} v(\beta)=0,
\end{aligned}
$$

因此有 $A_{\gamma_{k}} v \in V$. 这表明对 $k=0,1, \cdots, m-1, V$ 是 $A_{\gamma_{k}}$ 的不变线性空间.

以下证明定理的必要性. 因为对 $j=1,2, \cdots, r$ 及 $k=0,1, \cdots, m-1$, 有 $e_{j} \nabla_{-\gamma_{k}} \delta \in V$, 因 此 $A_{\gamma_{0}}\left(e_{j} \nabla_{-\gamma_{k}}\right) \delta \in V$. 这样对 $k=0,1, \cdots, m-1$, 有

$$
e_{1}^{\mathrm{T}} \sum_{\alpha \in \mathbb{Z}^{s}} a\left(M \alpha+\gamma_{k}\right)=e_{1}^{\mathrm{T}} \sum_{\alpha \in \mathbb{Z}^{s}} a(M \alpha) .
$$

由 (0.5) 式得到

$$
m e_{1}^{\mathrm{T}}=e_{1}^{\mathrm{T}} \sum_{\alpha \in \mathbb{Z}^{s}} a(\alpha)=e_{1}^{\mathrm{T}} \sum_{k=0}^{m-1} \sum_{\beta \in \mathbb{Z}^{s}} a\left(M \beta+\gamma_{k}\right) .
$$

以上的等式证明了定理的必要性.

注 1.2 当 $s=1, M=2$ 及 $1 \leqslant p \leqslant \infty$ 时, Jia 等在文献 [2] 中得到了类似的结论. 


\section{2 细分格式的收敛性和联合谱半径}

设 $\mathcal{A}$ 是由定义在有限维向量空间 $W$ 上的有限个线性算子构成的集合. 用 $\|\cdot\|$ 表示 $W$ 中 的范数. $\mathcal{A}$ 中线性算子的范数由下式给出:

$$
\|A\|:=\max _{\|v\|=1}\{\|A v\|\} .
$$

如果 $W$ 的子空间是 $\mathcal{A}$ 中每个算子的不变子空间，则称它是 $\mathcal{A}$ 不变的. 对 $w \in W$, 称所有 $W$ 中包含 $w$ 的 $\mathcal{A}$ 的不变子空间的交集为由 $w$ 生成的最小的 $\mathcal{A}$ 的不变子空间，记为 $W(w)$. 设 $n$ 为正整数, $\mathcal{A}$ 的 Descartes 幕

$$
\mathcal{A}^{n}=\left\{\left(A_{1}, \cdots, A_{n}\right): A_{1}, \cdots, A_{n} \in \mathcal{A}\right\} .
$$

当 $n=0$ 时, 记 $\mathcal{A}^{0}$ 为集合 $\{I\}$, 其中 $I$ 为定义在 $W$ 上的恒等映射.

设

$$
\left\|\mathcal{A}^{n}\right\|_{\infty}:=\max \left\{\left\|A_{1} \cdots A_{n}\right\|:\left(A_{1}, \cdots, A_{n}\right) \in \mathcal{A}^{n}\right\} .
$$

一致联合谱半径的定义由下式给出:

$$
\rho_{\infty}(\mathcal{A}):=\lim _{n \rightarrow \infty}\left\|\mathcal{A}^{n}\right\|_{\infty}^{1 / n} .
$$

Rota 和 Strang 在文献 [16] 中引入一致联合谱半径的概念. 文献 [17] 将一致联合谱半径应 用于小波理论的研究中.

设

$$
\left\|\mathcal{A}^{n}\right\|_{p}:=\left(\sum_{\left(A_{1}, \cdots, A_{n}\right) \in \mathcal{A}^{n}}\left\|A_{1} \cdots A_{n}\right\|^{p}\right)^{1 / p}, \quad 0<p<\infty .
$$

$p$ - 范数联合谱半径的定义由下式给出:

$$
\rho_{p}(\mathcal{A})=\lim _{n \rightarrow \infty}\left\|\mathcal{A}^{n}\right\|_{p}^{1 / n} .
$$

众所周知上述极限存在并且如下等式成立:

$$
\lim _{n \rightarrow \infty}\left\|\mathcal{A}^{n}\right\|_{p}^{1 / n}=\inf _{n \in \mathbb{N}}\left\|\mathcal{A}^{n}\right\|_{p}^{1 / n} .
$$

Wang 在文献 [18] 中引入 1- 范数联合谱半径的定义. 当 $1<p<\infty$ 时, Jia 在文献 [9] 中 引入 $p$ - 范数联合谱半径的定义. 当 $0<p<1$ 时, 这个概念也出现在文献 $[15,19]$ 中. 我们知 道利用定义来计算 $p$ - 范数联合谱半径是十分困难的. 当 $p$ 为偶数时, $\mathrm{Zhou}^{[19]}$ 得到了一个有 效的计算公式.

当 $1 \leqslant p<\infty, w \in W$ 时, 记

$$
\left\|\mathcal{A}^{n} w\right\|_{p}:=\left(\sum_{\left(A_{1}, \cdots, A_{n}\right) \in \mathcal{A}^{n}}\left\|A_{1} \cdots A_{n} w\right\|^{p}\right)^{1 / p} .
$$

当 $p=\infty$ 时, 记

$$
\left\|\mathcal{A}^{n} w\right\|_{\infty}:=\max \left\{\left\|A_{1} \cdots A_{n} w\right\|:\left(A_{1}, \cdots, A_{n}\right) \in \mathcal{A}^{n}\right\} .
$$

设 $1 \leqslant p \leqslant \infty$, 用 $\left(l_{p}\left(\mathbb{Z}^{s}\right)\right)^{r \times d}$ 表示所有满足 $\|c\|_{p}<\infty$ 的 $r \times d$ 矩阵值序列的全体, 其中 $c=\left(c_{j l}(\alpha)\right)_{1 \leqslant j \leqslant r, 1 \leqslant l \leqslant d}$, 当 $1 \leqslant p<\infty$ 时,

$$
\|c\|_{p}=\left(\sum_{l=1}^{d} \sum_{j=1}^{r} \sum_{\alpha \in \mathbb{Z}^{s}}\left|c_{j l}(\alpha)\right|^{p}\right)^{1 / p}
$$


当 $p=\infty$ 时, $\|c\|_{\infty}$ 表示 $\max _{1 \leqslant j \leqslant r, 1 \leqslant l \leqslant d}\left|c_{j l}\right|$ 在 $\mathbb{Z}^{s}$ 上的上确界.

以下假设 $\mathcal{A}=\left\{A_{\varepsilon}: \varepsilon \in B\right\}, W=\left(l_{0}\left(\mathbb{Z}^{s}\right)\right)^{r}$, 其中 $A_{\varepsilon}$ 由 (1.1) 式给出. 用 $\Omega$ 表示集合 $\left\{\alpha \in \mathbb{Z}^{s}: a(\alpha) \neq 0\right\}$.

由文献 $[1,15]$ 知

$$
\left\|\mathcal{A}^{n} w\right\|_{p}=\left\|a_{n} * w\right\|_{p}, \quad w \in\left(l_{0}\left(\mathbb{Z}^{s}\right)\right)^{r}, \quad 1 \leqslant p \leqslant \infty,
$$

其中 $a_{n} \in\left(l_{0}\left(\mathbb{Z}^{s}\right)\right)^{r \times r}$ 和 $w \in\left(l_{0}\left(\mathbb{Z}^{s}\right)\right)^{r}$ 的离散卷积 $a_{n} * w$ 由下式确定:

$$
a_{n} * w(\alpha)=\sum_{\beta \in \mathbb{Z}^{s}} a_{n}(\alpha-\beta) w(\beta), \quad \alpha \in \mathbb{Z}^{s},
$$

由文献 $[1,10]$ 知, 如果 $Y$ 是 $W$ 的子空间 $W_{1}$ 的一组基, 并且 $W_{1}$ 是 $\mathcal{A}$ 不变的, 则存在独 立于 $n$ 的正常数 $C$, 使得

记

$$
\left\|\left.\mathcal{A}^{n}\right|_{W_{1}}\right\|_{p} \leqslant C \max _{y \in Y}\left\|\mathcal{A}^{n} y\right\|_{p} \leqslant\left. C \max _{y \in Y}\left\|\left.\mathcal{A}^{n}\right|_{W_{1}}\right\|\right|_{p}\|y\| .
$$

$$
K:=\sum_{n=1}^{\infty} M^{-n} G
$$

其中集合 $G$ 由下式给出：

$$
G:=(\Omega-B) \cup\{0\}+M[-1,1]^{s} .
$$

用 $l(K)$ 表示所有支集为 $K$ 的序列构成的线性空间，易知 $(l(K))^{r}$ 是 $A_{\varepsilon}$ 不变的，其中 $\varepsilon \in B$.

记

$$
U:=V \cap(l(K))^{r},
$$

其中 $V$ 由 (1.7) 式给出, 则 $U$ 是一个有限维向量空间, 并且 $U$ 包含 $e_{1} \nabla_{j} \delta, j=1,2, \cdots, r$.

以下定理给出了与 $a$ 及 $M$ 有关的细分格式在 $\left(L_{p}\left(\mathbb{R}^{s}\right)\right)^{r}(1 \leqslant p \leqslant \infty)$ 中收敛的充分必要条 件.

定理 2.1 设 $\mathcal{A}=\left\{A_{\varepsilon}: \varepsilon \in B\right\}$, 其中 $A_{\varepsilon}$ 由 (1.1) 式给出. 又设 $M$ 是一般的扩张矩阵, $a \in\left(l_{0}\left(\mathbb{Z}^{s}\right)\right)^{r \times r}$, 使得由 $(0.4)$ 式确定的 $H(0)$ 具有 $(0.5)$ 式的形式. 记 $m:=|\operatorname{det} M|$, 则与 $a$ 及 $M$ 有关的细分格式在 $\left(L_{p}\left(\mathbb{R}^{s}\right)\right)^{r}(1 \leqslant p \leqslant \infty)$ 中收敛的充分必要条件是

(a) $e_{1}^{\mathrm{T}} \sum_{\alpha \in \mathbb{Z}^{\mathrm{s}}} a\left(M \alpha+\gamma_{k}\right)=e_{1}^{\mathrm{T}}, \quad k=0,1, \cdots, m-1$,

(b) $\rho_{p}\left(\left.\mathcal{A}\right|_{U}\right)<m^{1 / p}$.

证 只证明 $1 \leqslant p<\infty$ 时的情形, 因为 $p=\infty$ 时的情形是类似的. 首先证明定理的必要 性. 由定理 1.1 和 1.2 及以上的讨论, 知 (a) 是与 $a$ 及 $M$ 有关的细分格式在 $\left(L_{p}\left(\mathbb{R}^{s}\right)\right)^{r}(1 \leqslant p \leqslant \infty)$ 中收玫的必要条件. 为了证明 (b) 是必要的, 利用文献 [2] 定理 5.3 中证明的一些想法. 因为 $e_{1} \nabla_{j} \delta \in U, j=1,2, \cdots, r$, 因此 $U$ 中的向量是由向量 $e_{1} \nabla_{j} \delta_{\beta}, e_{2} \delta_{\beta}, \cdots, e_{r} \delta_{\beta}(j=1,2, \cdots, r, \beta \in$ $\left.\mathbb{Z}^{s}\right)$ 的线性组合生成的. 由 (2.4) 式得到, 对 $j=1,2, \cdots, s$ 和 $l=1,2, \cdots, r$ 及任意的 $\beta \in \mathbb{Z}^{s}$, 有

$$
\left\|\mathcal{A}^{n}\left(e_{1} \nabla_{j} \delta_{\beta}\right)\right\|_{p}=\left\|a_{n} *\left(e_{1} \nabla_{j} \delta\right)\right\|_{p}
$$

及

$$
\left\|\mathcal{A}^{n}\left(e_{l} \delta_{\beta}\right)\right\|_{p}=\left\|a_{n} *\left(e_{l} \delta\right)\right\|_{p} .
$$

设 $f=e_{l} \varphi_{0}$, 其中 $1 \leqslant l \leqslant r, \varphi_{0}$ 由 (1.6) 式确定. 利用 (0.8) 式, 得到

$$
Q_{a}^{n} f=\sum_{\alpha \in \mathbb{Z}^{s}} a_{n}(\alpha) f\left(M^{n} \cdot-\alpha\right)=\sum_{\alpha \in \mathbb{Z}^{s}}\left[a_{n}(\alpha) e_{l}\right] \varphi_{0}\left(M^{n} \cdot-\alpha\right),
$$


因此

$$
\begin{aligned}
\left\|Q_{a}^{n} f\right\|_{p}^{p} & =m^{-n} \sum_{\beta \in \mathbb{Z}^{s}} \int_{[0,1)^{s}+\beta}\left|\sum_{\alpha \in \mathbb{Z}^{s}}\left[a_{n}(\alpha) e_{l}\right] \varphi_{0}(x-\alpha)\right|^{p} d x \\
& =m^{-n}|| a_{n} e_{l}\left\|_{p}^{p}=m^{-n}|| a_{n}\left(e_{l} \delta\right)\right\|_{p}^{p},
\end{aligned}
$$

其中向量值函数 $\psi=\left(\psi_{1}, \cdots, \psi_{r}\right)^{\mathrm{T}} \in\left(L_{p}\left(\mathbb{R}^{s}\right)\right)^{r},|\psi|^{p}=\sum_{j=1}^{r}\left|\psi_{j}(x)\right|^{p}$. 设 $2 \leqslant l \leqslant r$, 向量 $e_{1} \varphi_{0}$ 和 $e_{1} \varphi_{0}+e_{l} \varphi_{0}$ 都满足 $(0.13)$ 式, 因此 $Q_{a}^{n}\left(e_{1} \varphi_{0}\right)$ 和 $Q_{a}^{n}\left(e_{1} \varphi_{0}+e_{l} \varphi_{0}\right)$ 在 $\left(L_{p}\left(\mathbb{R}^{s}\right)\right)^{r}(1 \leqslant p \leqslant \infty)$ 均收玫到方程 $(0.1)$ 的规范化解 $\varphi$, 从而对 $l=2, \cdots, r$, 有

$$
\lim _{n \rightarrow \infty} m^{-n / p}\left\|a_{n} *\left(e_{l} \delta\right)\right\|_{p}=0 .
$$

对 $f=e_{1} \varphi_{0}$, 有

$$
Q_{a}^{n} f-Q_{a}^{n} f\left(\cdot-M^{-n} e_{j}\right)=\sum_{\alpha \in \mathbb{Z}^{s}} \nabla_{j} a_{n}(\alpha) f\left(M^{n} \cdot-\alpha\right)=\sum_{\alpha \in \mathbb{Z}^{s}}\left[\nabla_{j} a_{n}(\alpha) e_{1}\right] \varphi_{0}\left(M^{n} \cdot-\alpha\right),
$$

因此

$$
\left\|Q_{a}^{n} f-Q_{a}^{n} f\left(\cdot-M^{-n} e_{j}\right)\right\|_{p}=m^{-n / p}\left\|\nabla_{j} a_{n} e_{1}\right\|_{p}=m^{-n / p}\left\|a_{n} *\left(e_{1} \nabla_{j} \delta\right)\right\|_{p} .
$$

然而, 由三角不等式得到

$$
\left\|Q_{a}^{n} f-Q_{a}^{n} f\left(\cdot-M^{-n} e_{j}\right)\right\|_{p} \leqslant 2\left\|Q_{a}^{n} f-\varphi\right\|_{p}+\left\|\varphi-\varphi\left(\cdot-M^{-n} e_{j}\right)\right\|_{p},
$$

这样

$$
\lim _{n \rightarrow \infty} m^{-n / p}\left\|a_{n} *\left(e_{1} \nabla_{j} \delta\right)\right\|_{p}=0, \quad j=1,2, \cdots, s
$$

注意到

$$
\rho_{p}\left(\left.\mathcal{A}\right|_{U}\right)=\lim _{n \rightarrow \infty}\left\|\left.\mathcal{A}^{n}\right|_{U}\right\|_{p}^{1 / n}=\inf _{n \geqslant 1}\left\|\left.\mathcal{A}^{n}\right|_{U}\right\|_{p}^{1 / n} .
$$

利用上述等式及 $(2.6) \sim(2.10)$ 式, 我们证明了定理的必要性.

下面证明定理的充分性. 充分性的证明依赖于定理 1.2 和文献 [1] 中引理 4.3. 在 $\left(L_{p}\left(\mathbb{R}^{s}\right)\right)^{r}$ 中选择一个紧支集向量值函数 $f$, 使得 $f$ 满足 $(0.13)$ 式. 估计 $\left\|Q_{a}^{n+1} f-Q_{a}^{n} f\right\|_{p}$, 由 $(0.8)$ 式有

$$
Q_{a}^{n+1} f-Q_{a}^{n} f=Q_{a}^{n} f_{0}=\sum_{\alpha \in \mathbb{Z}^{s}} a_{n}(\alpha) f_{0}\left(M^{n} x-\alpha\right),
$$

其中 $f_{0}=Q_{a} f-f$. 注意到

$$
\left\|Q_{a}^{n} f_{0}\right\|_{p}^{p}=\int_{\mathbb{R}^{s}}\left|\left(Q_{a}^{n} f_{0}\right)(x)\right|^{p} d x=\sum_{\beta \in \mathbb{Z}^{s}} \int_{M^{-n}\left([0,1)^{s}+\beta\right)}\left|\left(Q_{a}^{n} f_{0}\right)(x)\right|^{p} d x .
$$

对上述积分做变量替换 $x=M^{-n}(y+\beta)$, 由 $(2.4)$ 式有

$$
\left\|Q_{a}^{n} f_{0}\right\|_{p}^{p}=m^{-n} \int_{[0.1)^{s}} \sum_{\beta \in \mathbb{Z}^{s}}\left|\sum_{\alpha \in \mathbb{Z}^{s}} a_{n}(\alpha) f_{0}(y+\beta-\alpha)\right|^{p} d y=m^{-n} \int_{[0,1)^{s}}\left\|a_{n} * u_{y}\right\|_{p}^{p} d y,
$$

其中对 $\alpha \in \mathbb{Z}^{s}$ 及 $y \in[0,1)^{s}$, 有 $u_{y}(\alpha)=f_{0}(y+\alpha)$. 因为 $f_{0} \in\left(L_{p}\left(\mathbb{R}^{s}\right)\right)^{r}$, 并且是紧支集的, 因此 对所有的 $y \in[0,1)^{s}$, 有 $u_{y} \in\left(l_{0}\left(\mathbb{Z}^{s}\right)\right)^{r}$. 这样,

$$
\int_{[0,1)^{s}}|| u_{y} \|_{p}^{p} d y=\int_{[0,1)^{s}} \sum_{\alpha \in \mathbb{Z}^{s}}\left|f_{0}(y+\alpha)\right|^{p} d y=\int_{\mathbb{R}^{s}}\left|f_{0}(y)\right|^{p} d y .
$$


由条件 (a) 和 (0.13) 式, 得到对任意的 $y \in[0,1)^{s}$,

$$
\begin{aligned}
e_{1}^{\mathrm{T}} \sum_{\alpha \in \mathbb{Z}^{s}} u_{y}(\alpha) & =e_{1}^{\mathrm{T}} \sum_{\alpha \in \mathbb{Z}^{s}} \sum_{\beta \in \mathbb{Z}^{s}} a(\beta) f(M y+M \alpha-\beta)-e_{1}^{\mathrm{T}} \sum_{\alpha \in \mathbb{Z}^{s}} f(y+\alpha) \\
& =\sum_{\gamma \in \mathbb{Z}^{s}}\left[e_{1}^{\mathrm{T}} \sum_{\alpha \in \mathbb{Z}^{s}} a(M \alpha-\gamma)\right] f(M y+\gamma)-e_{1}^{\mathrm{T}} \sum_{\alpha \in \mathbb{Z}^{s}} f(y+\alpha)=0,
\end{aligned}
$$

因此对任意的 $y \in[0,1)^{s}$, 有 $u_{y} \in V$, 这里 $V$ 由 (1.7) 式确定. 如果对任意的 $y \in[0,1)^{s}$, 有 $u_{y} \in U=V \cap(l(K))^{r}$, 则由 (2.4) 式, 能够找到一个正数 $\rho<m^{1 / p}$, 使得对任意的 $y \in[0,1)^{s}$ 有

$$
\left\|a_{n} * u_{y}\right\|_{p}=\left\|\mathcal{A}^{n} u_{y}\right\|_{p} \leqslant C \rho^{n}\left\|u_{y}\right\|_{p}, \quad n=1,2, \cdots,
$$

其中 $C$ 是一个独立于 $n$ 和 $y$ 的正常数. 由 $(2.11) \sim(2.13)$ 式得到

$$
\left\|Q_{a}^{n} f_{0}\right\|_{p} \leqslant C\left(m^{-1 / p} \rho\right)^{n}\left\|f_{0}\right\|_{p} .
$$

这表明 $\left(Q_{a}^{n} f\right)_{n=1,2, \cdots}$ 是 $\left(L_{p}\left(\mathbb{R}^{s}\right)\right)^{r}(1 \leqslant p<\infty)$ 中的一个 Cauchy 序列, 因此, $\left(Q_{a}^{n} f\right)_{n=1,2, \cdots}$ 在 $\left(L_{p}\left(\mathbb{R}^{s}\right)\right)^{r}(1 \leqslant p<\infty)$ 中收敛.

如果存在 $[0,1)^{s}$ 的一个具有正测度的子集 $K_{0}$, 使得当 $y \in K_{0}$ 时, 有 $u_{y} \notin U=V \cap(l(K))^{r}$. 利用文献 [1] 中的引理 4.3 , 也能找到一个正数 $\rho<m^{1 / p}$, 使得对任意的 $y \in[0,1)^{s}$ 有

$$
\left\|a_{n} * u_{y}\right\|_{p}=\left\|\mathcal{A}^{n} u_{y}\right\|_{p} \leqslant C_{1} \rho^{n}\left\|u_{y}\right\|_{p}, \quad n=1,2, \cdots,
$$

其中 $C_{1}$ 是只依赖于集 $K_{0}$ 的正常数.

由 (2.11) 和 $(2.12)$ 及 $(2.14)$ 式得到

$$
\left\|Q_{a}^{n} f_{0}\right\|_{p}=m^{-n / p}\left(\int_{[0,1)^{s}}\left\|a_{n} * u_{y}\right\|_{p}^{p} d y\right)^{1 / p} \leqslant C_{1}\left(m^{-1 / p} \rho\right)^{n}\left\|f_{0}\right\|_{p},
$$

因此 $\left(Q_{a}^{n} f\right)_{n=1,2, \cdots}$ 也在 $\left(L_{p}\left(\mathbb{R}^{s}\right)\right)^{r}(1 \leqslant p<\infty)$ 中收玫. 证毕.

注 2.1 在非齐次的情形, Zhou ${ }^{[15]}$ 研究了细分格式在 $\left(L_{p}\left(\mathbb{R}^{s}\right)\right)^{r}(1 \leqslant p \leqslant \infty)$ 中的收玫 性. 然而, 在齐次的情形下, 研究细分格式在 $\left(L_{p}\left(\mathbb{R}^{s}\right)\right)^{r}(1 \leqslant p \leqslant \infty)$ 中的收玫性完全不同于非 齐次的情形. 在非齐次的情形下, 初始向量值函数的选择依赖于非齐次项. 在齐次的情形下, 初始向量值函数必须是满足 (0.13) 式的一类向量值函数 (参见文献 $[1,9,10,12,20 \sim 23]$ ).

\section{3 细分格式的 $L_{2}$ - 收敛性}

利用定义 (2.2) 来计算 $p$ - 范数联合谱半径是十分困难的. 当 $p$ 为偶数时, Zhou 在文献 [19] 中得到了一个有效的计算公式. 当 $p=2$ 时, 计算联合谱半径的问题归结为计算某一个确定矩 阵的谱半径的问题. 本节研究细分格式在 $\left(L_{2}\left(\mathbb{R}^{s}\right)\right)^{r}$ 中的收玫性. 我们的刻画依赖于作者的先 前工作 ${ }^{1)}$. 为了本节的需要, 我们引入一些相关的结果.

设 $a \in\left(l_{0}\left(\mathbb{Z}^{s}\right)\right)^{r \times r}, M$ 是一般的扩张矩阵. 用下式定义由 $\left(l_{0}\left(\mathbb{Z}^{s}\right)\right)^{r \times r}$ 到 $\left(l_{0}\left(\mathbb{Z}^{s}\right)\right)^{r \times r}$ 的线 性算子 $F_{a}^{[4]}$ :

$$
\widetilde{F_{a} w}\left(e^{-\mathrm{i} \xi}\right)=\frac{1}{m^{2}} \sum_{i=1}^{m} \tilde{a}\left(e^{-\mathrm{i}\left(M^{\mathrm{T}}\right)^{-1}\left(\xi+2 \pi d^{i}\right)}\right) \tilde{w}\left(e^{-\mathrm{i}\left(M^{\mathrm{T}}\right)^{-1}\left(\xi+2 \pi d^{i}\right)}\right) \tilde{a}\left(e^{-\mathrm{i}\left(M^{T}\right)^{-1}\left(\xi+2 \pi d^{i}\right)}\right)^{*},
$$

1) Li S. Characterizations of smoothness of multivariate refinable functions and convergence of cascade algorithms of nonhomogeneous refinement equations. Advances in Comp Math (待发表) 
其中 $w \in\left(l_{0}\left(\mathbb{Z}^{s}\right)\right)^{r \times r}, \tilde{a}\left(e^{-\mathrm{i}\left(M^{\mathrm{T}}\right)^{-1}\left(\xi+2 \pi d^{i}\right)}\right)^{*}$ 为 $\tilde{a}\left(e^{-\mathrm{i}\left(M^{\mathrm{T}}\right)^{-1}\left(\xi+2 \pi d^{i}\right)}\right)$ 的共轭转置, $\xi=\left(\xi_{1}, \cdots, \xi_{s}\right) \in$ $\mathbb{R}^{s}, \tilde{b}(z):=\sum_{\alpha \in \mathbb{Z}^{s}} b(\alpha) z^{\alpha}, z \in \mathbb{C}^{s} \backslash\{0\}$ 表示向量 $b \in\left(l_{0}\left(\mathbb{Z}^{s}\right)\right)^{r \times r}$ 的符号.

作者先前的工作证明了如下结论: 设 $v \in\left(l_{0}\left(\mathbb{Z}^{s}\right)\right)^{r}$, 则

$$
\lim _{n \rightarrow \infty}\left\|a_{n} * v\right\|_{2}^{2 / n}=m \rho\left(\left.F_{a}\right|_{W}\right),
$$

其中 $W$ 是由 $w$ 生成的最小的 $F_{a}$ 的不变子空间, $a_{n}$ 由 $(0.9)$ 式给出, $w(\beta):=\sum_{\gamma \in \mathbb{Z}^{s}} v(\beta+$ $\gamma) v(\gamma)^{*}, \beta \in \mathbb{Z}^{s}$.

设 $j=1, \cdots, s$, 差分算子 $\Delta_{j}$ 由下式给出:

$$
\Delta_{j} u:=2 u-u\left(\cdot-e_{j}\right)-u\left(\cdot+e_{j}\right), \quad u \in l_{0}\left(\mathbb{Z}^{s}\right) .
$$

以下是本节的主要结果:

定理 3.1 设 $\mathcal{A}=\left\{A_{\varepsilon}: \varepsilon \in B\right\}, a$ 和 $M$ 与定理 2.1 中一致. 又设 $F_{a}$ 是由 (3.1) 式确定的 线性算子, 则有

$$
\rho_{2}^{2}\left(\left.\mathcal{A}\right|_{V_{1}}\right)=m \rho\left(\left.F_{a}\right|_{V_{2}}\right)
$$

其中 $V_{1}$ 是由向量 $e_{1} \nabla_{j} \delta(j=1,2, \cdots, s), e_{2} \delta, \cdots, e_{r} \delta$ 生成的最小的 $\mathcal{A}$ 的不变子空间, $V_{2}$ 是由 向量 $e_{1} e_{1}^{\mathrm{T}} \Delta_{j} \delta, e_{2} e_{2}^{\mathrm{T}} \delta, \cdots, e_{r} e_{r}^{\mathrm{T}} \delta(j=1,2, \cdots, s)$ 生成的最小的 $F_{a}$ 的不变子空间, 因此与 $a$ 及 $M$ 有关的细分格式在 $\left(L_{2}\left(\mathbb{R}^{s}\right)\right)^{r}$ 中收敛的充分必要条件是

$$
\rho\left(\left.F_{a}\right|_{V_{2}}\right)<1 \text {. }
$$

证 因为 $V_{1}$ 是由向量 $e_{1} \nabla_{j} \delta(j=1,2, \cdots, s), e_{2} \delta, \cdots, e_{r} \delta$ 生成的最小的 $\mathcal{A}$ 的不变子空间, 利用 $(2.6) \sim(2.8)$ 式, 有

$$
\rho_{2}\left(\left.\mathcal{A}\right|_{V_{1}}\right)=\lim _{n \rightarrow \infty} \max \left\{\left\|a_{n} *\left(e_{1} \nabla_{j} \delta\right)\right\|_{2}^{1 / n}, 1 \leqslant j \leqslant s, \max _{2 \leqslant l \leqslant r}\left\{\left\|a_{n} *\left(e_{1} \nabla \delta\right)\right\|_{2}^{1 / n}\right\}\right\} .
$$

由 (3.2) 式得到

$$
\rho_{2}^{2}\left(\left.\mathcal{A}\right|_{V_{1}}\right)=m \rho\left(\left.F_{a}\right|_{V_{2}}\right) .
$$

当 $v=e_{1} \nabla_{j} \delta, e_{2} \delta, \cdots, e_{r} \delta$ 时, 对应的 $w$ 分别是 $e_{1} e_{1}^{\mathrm{T}} \Delta_{j} \delta, e_{2} e_{2}^{\mathrm{T}} \delta, \cdots, e_{r} e_{r}^{\mathrm{T}} \delta$. 令 $V_{2}$ 是由这些向量 生成的最小的 $F_{a}$ 的不变子空间. 由定理 2.1, 得到与 $a$ 及 $M$ 有关的细分格式在 $\left(L_{2}\left(\mathbb{R}^{s}\right)\right)^{r}$ 中 收敛的充分必要条件是

$$
\rho\left(\left.F_{a}\right|_{V_{2}}\right)<1
$$

证毕.

注 3.1 当 $s=1, M=2$ 以及 $r=1$ 时, Han 和 Jia 等分别在文献 $[2,10]$ 中建立了类似的 结果.

\section{参 考文献}

1 Chen D R, Jia R Q, Riemenschneider S D. Convergence of vector subdivision schemes in Sobolev spaces. Applied and Computational Harmonic Analysis, 2002, 12: 128 149

2 Jia R Q, Riemenschneider S D, Zhou D X. Vector subdivision schemes and multiple wavelets. Math Comp, 1998, 67: 1533 1563

3 Heil C, Colella D. Matrix refinement equations: existence and uniqueness. J Fourier Anal Appl, 1996, 2: $363 \sim 377$ 
4 Jiang Q T. Multivariate matrix refinement functions with arbitrary matrix dilation. Tran Amer Math Soc, 1999, 351: 2407 2438

5 Cavaretta A S, Dahmen W, Micchelli C A. Stationary subdivision. Memior Amer Math Soc, 1991, 93: 1 186

6 Goodman T N F, Lee S L. Convergence of cascade algorithms. In: Daehlen M, Lyche T, Schumaker L L, eds. Mathematical Methods for Curves and Surfaces II. Nashville: Vanderbilt University Press, $1998.191 \sim 212$

7 Strang G, Nguyen T. Wavelets and Filter Banks. Boston: Wellesley-Cambridge Press, 1996

8 Dyn N, Gregory J A, Levin D. Analysis of uniform binary subdivision schemes for cure design. Constr Approx, 1991, 23: 127 147

9 Jia R Q. Subdivision schemes in $L_{p}$ spaces. Advances in Comp Math, 1995, 3: 309 341

10 Han B, Jia R Q. Multivariate refinement equations and convergence of subdivision schemes. SIAM J Math Anal, 1998, 29: 1177 1199

11 Strang G. Eigenvalues of $(\downarrow 2) \mathrm{H}$ and convergence of cascade algorithm. IEEE Trans SP, 1996, 44: 233 238

12 Lawton W, Lee S L, Shen Z W. Convergence of multidimensional cascade algorithms. Numer Math, 1998, 78: $427 \sim 438$

13 Long R L, Mo Q. $L^{2}$-convergence of vector cascade algorithm. Approx Theory Appl, 1999, 15: 24 49

14 Shen Z W. Refinable function vectors. SIAM J Math Anal, 1998, 29: 235 250

15 Zhou D X. Norms concerning subdivision sequences and their applications in wavelets. Applied and Computationic Harmonic Analysis, 2001, 11: 329 346

16 Rota C C, Strang G. A note on the joint spectral radius. Indag Math, 1960, 22: 379 381

17 Daubechies I, Lagarias J C. Two-scale difference equations: II. Local regularity, infinite products of matrices and fractals. SIAM J Math Anal, 1992, 23: 1031 1079

18 Wang Y. Two-scale dilation equations and mean spectral radius. Random Comput Dynam, 1996, 4: 49 72

19 Zhou D X. The $p$-norm joint spectral radius for even integers. Methods and Applications of Analysis, 1998, 5: $39 \sim 54$

20 Ji R Q, Jiang Q T, Shen Z W. Convergence of cascade algorithms associated with nonhomogeneous refinement equations. Proc Amer Math Soc, 2001, 129: 415 427

21 Jia R Q. Convergence of vector subdivision schemes and construction of biorthogonal multiple wavelets. In: Advance in Wavelets (Hong Kong, 1997). Singapore: Springer, 1998. 199 227

22 Li S. Convergence of cascade algorithms in Sobolev spaces associated with inhomogeneous refinement equations. J Approx Theory, 2000, 104: 135 163

23 Strang G, Zhou D X. Inhomogeneous refinement equations. J Fourier Anal Appl, 1998, 4: 733 747 